\title{
Surface profiles of reflow microlenses under the influence of surface tension and gravity
}

\author{
Andreas Schilling \\ Roman Merz \\ Christian Ossmann \\ Hans Peter Herzig \\ Institute of Microtechnology \\ University of Neuchâtel \\ Rue A.-L. Breguet 2 \\ $\mathrm{CH}-2000$ Neuchâtel, Switzerland \\ E-mail: andreas.schilling@imt.unine.ch
}

\begin{abstract}
We present a finite-element method to calculate 3-D surface profiles of refractive microlenses fabricated by melting-resist technology (reflow technique). The geometry of the microlenses can be arbitrary. Surface tension and gravity are taken into account. Gravity can have an advantageous influence on the profile form, so that smaller focal spots can be achieved. A simple scaling law is given to estimate the influence of gravity on the profile form for given microlens parameters. We compared various theoretical and measured surface profiles of microlenses fabricated by melting-resist technology and found good agreement. Finally, the usefulness of this method for the design of refractive microstructures for smart masks is shown. () 2000 Society of Photo-Optical Instrumentation Engineers. [S0091-3286(00)03208-6]
\end{abstract}

Subject terms: refractive microlenses; surface profile modeling; finite-element method; surface tension; smart masks.

Paper 990251 received June 22, 1999; revised manuscript received Jan. 7, 2000; accepted for publication Feb. 16, 2000.

\section{Introduction}

Refractive microlenses are used in various applications such as miniaturized chemical detection systems, ${ }^{1}$ microlens projection lithography, ${ }^{2}$ and confocal microscopy. ${ }^{3} \mathrm{~A}$ common and well-known method to fabricate refractive microlenses is the melting-resist technology (reflow technique). ${ }^{1,4,5}$ Photoresist is deposited onto a substrate, which is coated with a resist base layer and afterwards structured using standard photolithographic techniques. Subsequently, the resist is melted in an oven and microlenses are formed due to the surface tension of the liquid resist. The fabrication process is illustrated in Fig. 1. This process is well developed, and spherical microlenses, for example, can be fabricated with diffraction-limited optical properties. ${ }^{6}$

The fabrication process is dominated by experimental experience. Little work so far has been dedicated to theoretical investigations and analysis of the surface profiles in terms of the basic underlying physical quantities, especially for arbitrary microlens geometries. In the work of Sheridan et al. ${ }^{7}$ the modeling of melted spherical microlens shapes is treated with a heuristic approach, while in the work of Erdmann ${ }^{8}$ the restrictions concern either the geometry of the structures, approximations for small radii of curvature, or the exclusion of gravity. For spherical or elliptical microlenses without gravitational influence, the theoretical profile forms can be determined analytically, while for arbitrary microlens geometries the surface profiles have to be evaluated with numerical methods. Examples for this kind of more complicated profile forms are cylindrical microlenses intersecting at $90 \mathrm{deg}$ to form a microlens corner structure. Such structures might be used in smart-mask lithography where micro-optical elements are used to print simple patterns into photoresist. ${ }^{9}$
In this paper we present a finite-element method to calculate 3-D surface profiles of microlenses with arbitrary geometry under the influence of surface tension and gravity. We demonstrate that for certain parameter combinations gravity can have an advantageous influence on the surface profile form of microlenses. We give a simple scaling law to estimate the influence of gravity on the surface profiles of melting resist microlenses. We compare theoretical profile forms with measured profiles of fabricated structures to demonstrate the applicability of this finiteelement method for the modeling of the profile forms for melting-resist microlenses. For the example of cylindrical microlenses intersecting at $90 \mathrm{deg}$, we show how theoretical predictions for the surface profiles can be used to improve the design of smart mask structures.

\section{Theory}

The model that we apply to calculate the surface profiles of melting-resist microlenses is based on the assumption that at a certain point during the melting-resist fabrication process the resist is a liquid with a fixed surface tension and that a generalized minimum surface is formed under the additional influence of gravity. For a complete description of the problem one has to specify the geometry of the resist borders, the resist height, the surface tension and mass density of the liquid photoresist, and the acceleration of gravity. Here we assume that during the melting process the borders of the resist structures remain fixed, which is experimentally well justified, at least for the fabrication process we used. With these parameters, the problem is specified and the solution is completely determined if one further assumes that the volume is conserved during the melting process. If one desired to take into account resistsolvent evaporation during the melting process, the fabrica- 




(b)

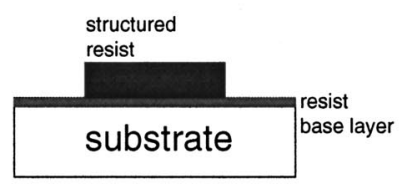

(c)

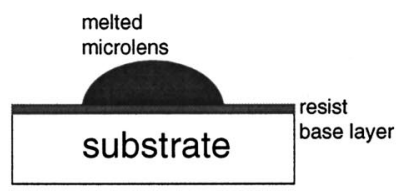

Fig. 1 Melting resist fabrication of microlenses: (a) Photoresist is deposited onto a substrate with a resist base layer and exposed to UV light through a chromium mask. (b) The exposed resist is processed, and free-standing resist structures are created. (c) The microlenses are formed during melting in an oven.

tion of evaporated solvent or the maximum microlens height after melting could be used as an additional parameter.

In the following, we present the basic equations for the generalized minimum-surface problem. The partial differential equation that describes the surface profile of a liquid with a surface tension $\sigma$ and mass density $\rho$ in an external potential $U$ is given by ${ }^{10}$

$\sigma\left[\frac{1}{R_{1}(x, y)}+\frac{1}{R_{2}(x, y)}\right]+\rho U(x, y, z)+\lambda=0$,

where $R_{1}(x, y)$ and $R_{2}(x, y)$ are the two principal radii of curvature, $\lambda$ is a constant, and $x$ and $y$ are Cartesian coordinates. In terms of the surface profile $z(x, y)$ we get for the radii of curvature

$$
\begin{aligned}
& \frac{1}{R_{1}(x, y)}+\frac{1}{R_{2}(x, y)} \\
& \quad=\operatorname{div}\left\{\frac{1}{\left[1+|\operatorname{grad} z(x, y)|^{2}\right]^{1 / 2}} \operatorname{grad} z(x, y)\right\} .
\end{aligned}
$$

The influence of gravity is described by the external potential $U=-g z$, where $g$ is the acceleration of gravity. From Eq. (1) we get finally for the surface profile $z(x, y)$ the differential equation

$\operatorname{div}\left\{\frac{1}{\left[1+|\operatorname{grad} z(x, y)|^{2}\right]^{1 / 2}} \operatorname{grad} z(x, y)\right\}-\frac{\rho g}{\sigma} z(x, y)+\frac{\lambda}{\sigma}$

$$
=0 \text {. }
$$

The geometry is illustrated in Fig. 2.

The coefficient $\rho g / \sigma$ defines a typical length scale $L_{c}$ $=(\sigma / \rho g)^{1 / 2}$, which is called the capillary length. In the following we replace the constant $\rho g / \sigma$ by $a$ and $\lambda / \sigma$ by $f$. Therefore, Eq. (3) takes the form

$\operatorname{div}\left\{\frac{1}{\left[1+|\operatorname{grad} z(x, y)|^{2}\right]^{1 / 2}} \operatorname{grad} z(x, y)\right\}-a z(x, y)+f=0$.
This elliptic partial differential equation was solved with a finite-element method, as implemented in the partial differential equation toolbox in MATLAB ${ }^{\mathrm{TM}}{ }^{11}$ according to the following scheme: First, the boundary of the microlens has to be specified. This boundary corresponds to the boundary of the resist cylinders before the melting process. There are no restrictions concerning the geometry of the boundary. Next, a mesh grid with an appropriate resolution has to be defined over the area of interest, the interior of the microlens boundaries. An appropriate resolution of the grid can always be found by using a set of mesh grids with increasing resolution while controlling the convergence of the obtained solutions.

Subsequently, the boundary conditions have to be specified. For the standard case it is clearly appropriate to use Dirichlet boundary conditions, where the solution $z$ for the surface profile is specified on the boundary. After melting the surface profile height is zero on the boundary; therefore the Dirichlet boundary condition becomes

$z(s(x, y)) \equiv 0$,

where $s(x, y)$ describes the boundary of the microlens. It can be useful for certain special cases to use other boundary conditions, like Neumann boundary conditions, where the derivative of the profile height is given on the boundary, or

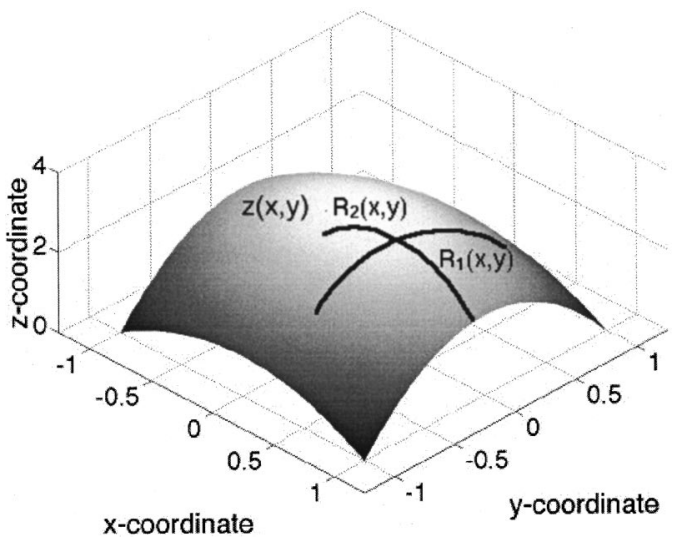

Fig. 2 Arbitrary surface $z(x, y)$ with the two principal radii of curvature $R_{1}(x, y)$ and $R_{2}(x, y)$. 


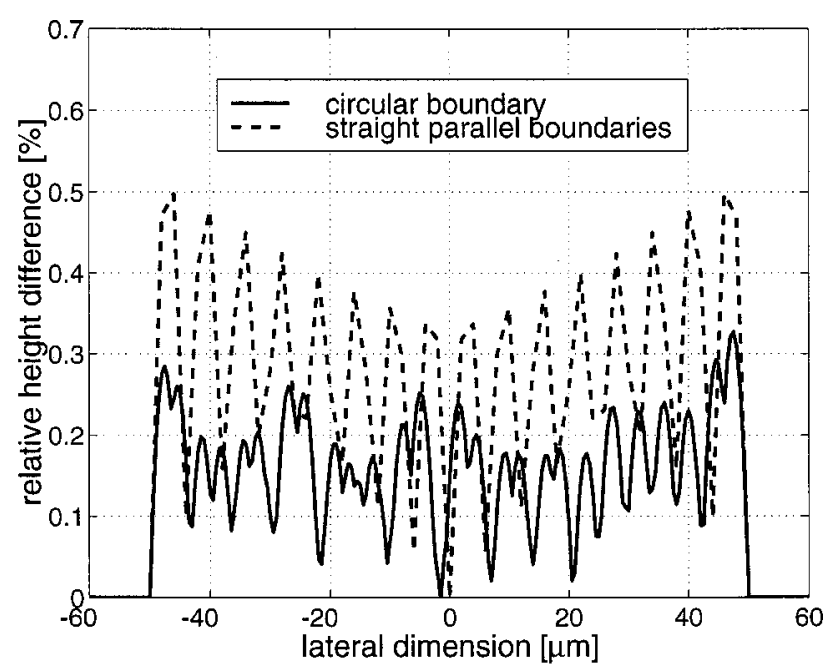

Fig. 3 Relative height difference between the analytical solution and the solution obtained by the modeling method. The solid line indicates a circular boundary, the dashed line straight parallel boundaries. Both cases are without gravity.

mixed boundary conditions, where a combination of $z$ and the derivative of $z$ is specified on the boundary.

The parameters $\sigma, \rho$, and $\lambda$, or equivalently $a$ and $f$, have to be specified. Of these, $\sigma$ and $\rho$ are material constants that depend on the photoresist used for the fabrication of the microlenses, and $\lambda$ has to be determined with the help of an additional parameter, such as the volume or maximum height of the final surface profile. Typical values for the mass density and surface tension of a photoresist are $\rho=1000 \mathrm{~kg} / \mathrm{m}^{3}$ and $\sigma=(2.5$ to 5$) \times 10^{-2} \quad \mathrm{~N} / \mathrm{m}$, respectively. ${ }^{8,12}$ Therefore, a typical value for the parameter $a$ is about $2 \times 10^{-7} \mu \mathrm{m}^{-2}$, which corresponds to a typical length scale of $L_{c} \approx 2 \mathrm{~mm}$.

In order to validate the modeling method, we compared the solutions obtained by it with the analytical solutions for two cases with simple boundaries without gravity: a circular boundary and straight parallel boundaries. In these cases the profiles are a spherical surface and a circular arc surface, respectively. For both cases the microlens heights were $10 \mu \mathrm{m}$ and the diameter or width was $100 \mu \mathrm{m}$. The relative height difference between the two solutions, as shown in Fig. 3, was in both cases smaller than $0.5 \%$. This remaining small difference is due to the grid of the finiteelement modeling method and could be reduced further by using a finer grid.

By rewriting Eq. (1) with the parameters $a$ and $f$, we obtain the equation

$\left[\frac{1}{R_{1}(x, y)}+\frac{1}{R_{2}(x, y)}\right]-a z(x, y)+f=0$.

If the linear dimensions and the surface profile height are scaled with an arbitrary dimensionless parameter $\beta$, we get

$x^{\prime}=\beta x$,

$y^{\prime}=\beta y$, (a)

(b)

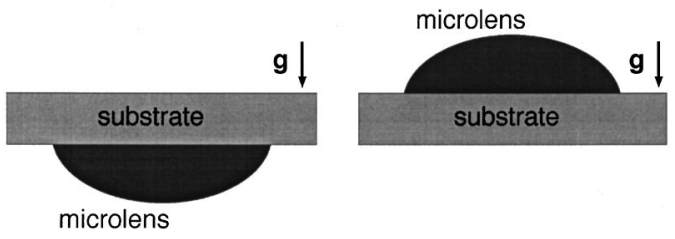

Fig. 4 Gravity $g$ acting on the melting photoresist during microlens formation: (a) hanging microlens $(a<0)$, (b) lying microlens $(a$ $>0)$.

$z^{\prime}(x, y)=\beta z(x, y)$,

and the radii of curvature scale as

$R_{i}^{\prime}=\beta R_{i}, \quad i=1,2$.

As a consequence, Eq. (6) remains invariant if at the same time the parameters $a$ and $f$ are scaled according to

$a^{\prime}=\frac{a}{\beta^{2}}$,

$f^{\prime}=\frac{f}{\beta}$.

The scaling property of Eq. (6) is employed in Sec. 3.

\section{Results and Discussion}

We have investigated the influence of the direction and strength of gravity on the surface profile form of spherical microlenses. The parameters $\rho, g$, and $\sigma$ appear in Eq. (4) in the combined form of the parameter $a=\rho g / \sigma$. For fixed values of mass density $\rho$ and surface tension $\sigma$, the variation of the parameter $a$ corresponds to a variation of gravity. We have to distinguish between two different cases. In the first case, gravity points from the substrate to the microlens surface (hanging microlenses), corresponding to $a$ $<0$. In the other case, gravity points from the microlens surface to the substrate (lying microlenses), corresponding to $a>0$. The two different configurations are displayed in Fig. 4

We have calculated for spherical microlenses with a diameter of $100 \mu \mathrm{m}$ and a typical resist cylinder height of 9 $\mu \mathrm{m}$ the surface profile forms as a function of the parameter $a$. Subsequently, we have calculated the corresponding Zernike polynomials of the surface and analyzed the profile forms with a commercial raytrace program (Raytrace $\left.6.1^{\mathrm{TM}}\right)$. We have evaluated the focal spot size for the microlenses with a refractive index of $n=1.5$, illuminated with a plane wave from the convex side. The focal spot was defined as the point of least confusion.

The results of the simulations are shown in Fig. 5. A significant influence on the spot size can be observed for parameter values $|a|>2 \times 10^{-5} \mu \mathrm{m}^{-2}$ in both cases of the direction of gravity. This value of $a$ corresponds to a capillary length of $L_{c} \approx 200 \mu \mathrm{m}$, which is on the order of the microlens diameter of $100 \mu \mathrm{m}$. For values $|a|>5$ 

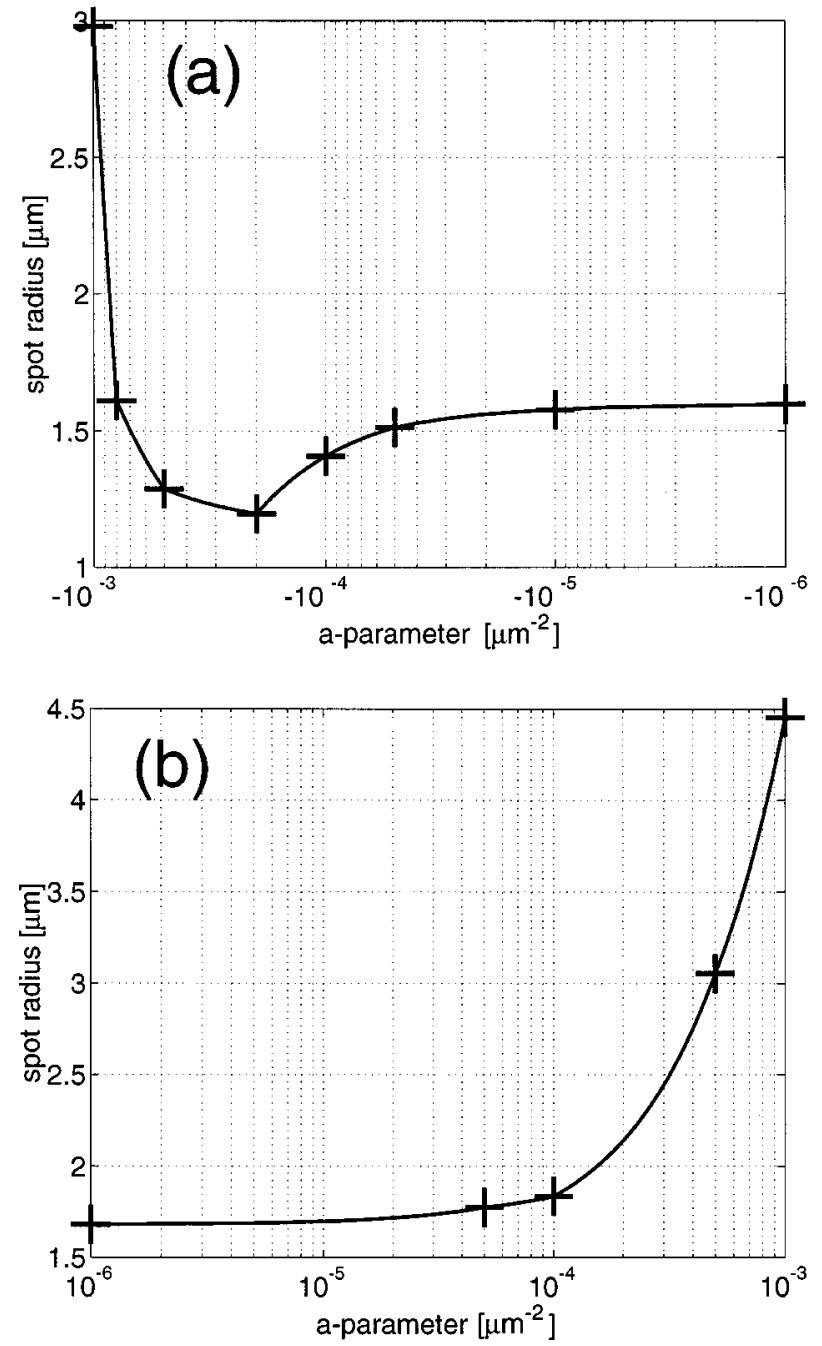

Fig. 5 Focal-spot size as a function of the parameter $a=\rho g / \sigma$ : (a) hanging microlens $(a<0)$, (b) lying microlens $(a>0)$.

$\times 10^{-4} \mu \mathrm{m}^{-2}$ the spot size changes dramatically. The behavior of the spot size is quite different for the two different directions of gravity. While for lying microlenses the focal-spot size increases continuously, for hanging microlenses a decrease in size with increasing $|a|$ is possible because of the reduction of spherical aberration by thickening at the center of the lens. The smallest spot is achieved for $|a| \approx 3 \times 10^{-4} \mu \mathrm{m}^{-2}$. The focal spot is $25 \%$ smaller than for a comparable microlens without gravitational influence in this configuration.

The scaling law introduced in Sec. 2 predicts that if the length scales for a given microlens geometry are changed by one order of magnitude, then the parameter $a=\rho g / \sigma$ has to be scaled by two orders of magnitude to reproduce an identical surface profile. As shown above, gravity starts to have an influence on the surface profile for $|a|>2$ $\times 10^{-5} \mu \mathrm{m}^{-2}$ for microlenses with diameter $100 \mu \mathrm{m}$. Therefore, one can conclude that, assuming a typical parameter value of $\alpha \approx 2 \times 10^{-7} \mu \mathrm{m}^{-2}$ for standard photoresist, gravitation will start to have a significant influence on the profile form for microlenses with diameters larger than about $1000 \mu \mathrm{m}$. Equivalently, if the parameter $a$ could be

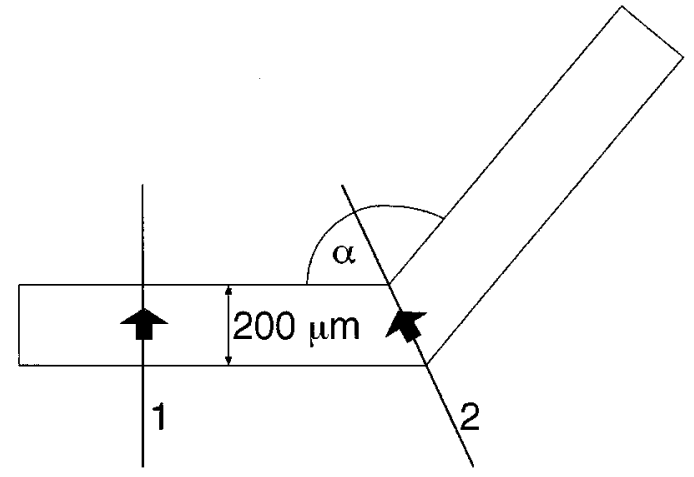

(a)



(b)

Fig. 6 Two cylindrical microlenses intersecting at an angle of $\alpha=135$ deg: (a) geometrical configuration, (b) theoretical and experimental profile curves for the cross sections 1 and 2 indicated in (a).

changed by two orders of magnitude, for example by using other materials or by increasing gravity with a centrifuge, the influence on the surface profile would become significant for diameters larger than about $100 \mu \mathrm{m}$.

Since the scaling law is valid in general, the conclusion about the point where gravity has a significant influence can be extended to arbitrary microlens structures where typical radii of curvature are comparable with the radii of curvature of the investigated spherical microlenses. For microlens structures with other typical radii of curvature, the critical value of the parameter $a$ for the onset of gravity influence can be determined by the scaling laws given in Eqs. (7) to (12).

We fabricated various test structures to verify the described finite-element modeling for microlens surface profiles with arbitrary geometry. The different geometries and the comparison of the theoretical and the experimental results are shown in Figs. 6-8. The microlenses were fabricated following the process described by Nussbaum et al. ${ }^{1}$ We used the photoresist AZ 4562 from Hoechst. We always calculated 3-D surface profiles and took cross sections for comparison with the measured curves. For the investigated microlenses the influence of gravity was neg- 


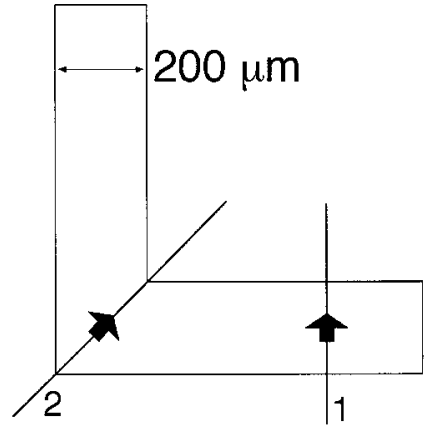

(a)

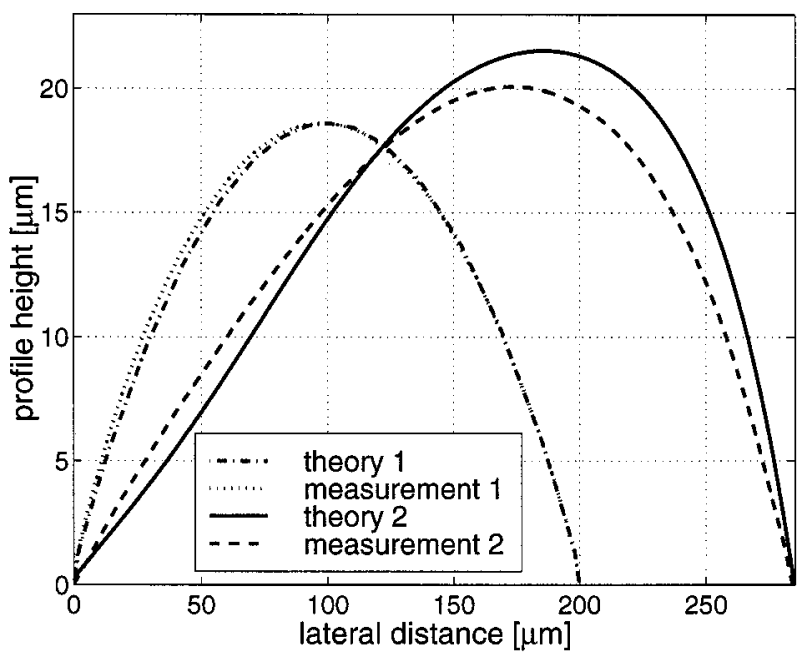

(b)

Fig. 7 Two cylindrical microlenses intersecting at an angle of $\alpha=90$ deg: (a) geometrical configuration, (b) theoretical and experimental profile curves for the cross sections 1 and 2 indicated in (a).

ligible; therefore all profile forms were calculated with $a$ $=0$. All experimental curves were measured with a profilometer (Tencor Instruments). The exact positions and directions of the measured curves were controlled with a microscope that allowed us to view the profilometer tip and the microstructures to be measured.

Figure 6(a) shows a microlens structure with two cylindrical microlenses intersecting at an angle of $135 \mathrm{deg}$. The comparison of the theoretical and experimental surface profiles for cross sections 1 and 2 as indicated in Fig. 6(a) is shown in Fig. 6(b). The agreement between calculated and measured profile forms is very good for cross section 1 . For cross section 2 the maximum height and the general profile form are well reproduced, while the lateral position of the maximum height is slightly displaced.

It is desirable for certain smart-mask applications to have connected, cylindrical microlenses that form a $90 \mathrm{deg}$ angle. We fabricated two different structures of connected cylindrical microlenses at an angle of $90 \mathrm{deg}$, shown in Figs. 7 and 8. In the layout of Fig. 7, the microlenses are directly connected, forming a sharp corner. In the layout of Fig. 8, the 90-deg corner is rounded, so that the microlens has a constant width. For these two structures we calculated and measured the profile forms and found again an experimental verification of the theoretical results, as expected.

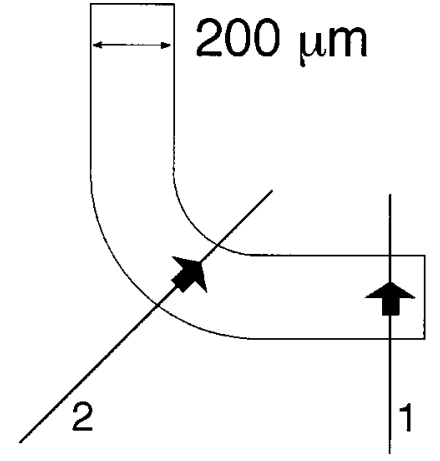

(a)

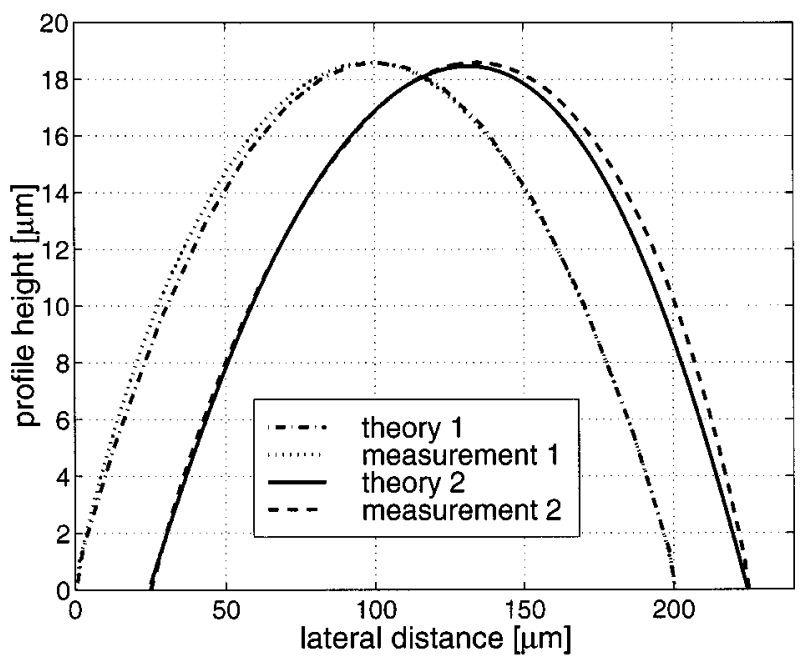

(b)

Fig. 8 Improved corner structure for two microlenses with a constant-width design: (a) geometrical configuration, (b) theoretical and experimental profile curves for the cross sections 1 and 2 indicated in (a).

Cross section 1 in Fig. 7 yields the same result as for the structure with $\alpha=135 \mathrm{deg}$, because it corresponds simply to the surface profile of a cylindrical microlens. The agreement between calculated and measured data for cross section 2 is satisfactory. However, the absolute profile height is slightly underestimated and the theoretical profile shows a slight deviation from the measured one. For both cross sections displayed in Fig. 8, we found excellent agreement between theoretical and experimental profiles.

The constant-width structure in Fig. 8, with a difference between interior and exterior radii of curvature equal to the width of the structure, has the expected property that the microlens height is constant and that the cross-sectional profile is identical for the whole microlens. The focal line is therefore produced in the same plane along the whole structure. Therefore this structure would be the most appropriate for smart-mask applications, where patterns in the focal plane have to be printed into resist. The fact that the agreement between theoretical and experimental surface profiles was better for smooth structures than for structures having sharp corners was also confirmed by other experiments. 


\section{Conclusions}

We have presented a finite-element method to determine theoretically the 3-D surface profiles of microlenses fabricated by melting-resist technology for arbitrary microlens geometries. For spherical microlenses the influence of gravity on the profile form was calculated and the optical properties of the resulting profile forms were analyzed with a commercial raytrace program. We found that for hanging microlenses a better focal spot can be achieved than for lying microlenses. With the help of a simple scaling law, we derived a rule of thumb to decide when gravity has a nonnegligible influence on the surface profiles of microlenses. We compared theoretical and experimental results for the surface profiles of different structures and found good agreement. For cylindrical microlenses intersecting at $90 \mathrm{deg}$, we demonstrated the utility of this modeling method for the design of nonstandard microlenses for smart-mask applications.

\section{Acknowledgments}

This research was supported by the Swiss Priority Program Optique II.

\section{References}

1. P. Nussbaum, R. Völkel, H. P. Herzig, M. Eisner, and S. Haselbeck, "Design, fabrication and testing of microlens arrays for sensors and microsystems," Pure Appl. Opt. 6, 617-636 (1997).

2. R. Völkel, H. P. Herzig, P. Nussbaum, and R. Dändliker, "Microlens array imaging system for photolithography," Opt. Eng. 35, 33233330 (1996).

3. M. Eisner, N. Lindlein, and J. Schwider, "Confocal microscopy with a refractive microlens-pinhole array," Opt. Lett. 23, 748-749 (1998).

4. M. C. Hutley, "The manufacture and testing of microlens arrays," Proc. SPIE 1319, 492-493 (1990)

5. D. Daly, R. F. Stevens, M. C. Hutley, and N. Davies, "The manufacture of microlenses by melting photoresist," J. Meas. Sci. Technol. 1 759-766 (1990)

6. P. Nussbaum, R. Völkel, H. P. Herzig, and R. Dändliker, "Microoptics for sensor applications,"' Proc. SPIE 2783, 55-63 (1996).

7. J. T. Sheridan, J. Schwider, N. Streibl, S. Haselbeck, M. Eisner, M. Heissmeier, and O. Falkenstörfer, "Modelling and measurement of melted microlens shapes," Optik (Stuttgart) 97, 174-180 (1994).

8. L. Erdmann, "Experimentelle und theoretische Untersuchungen zur Herstellung und Charakterisierung refraktiver Mikrolinsen," $\mathrm{PhD}$ Thesis, Universität Jena, Germany (1998).

9. R. Völkel, H. P. Herzig, P. Nussbaum, P. Blattner, R. Dändliker, E. Cullmann, and W. B. Hugle, "Microlens lithography and smart masks," Microelectron. Eng. 35, 513-516 (1997).

10. A. Budo, Theoretische Mechanik, 11th ed., VEB Deutscher Verlag der Wissenschaften, Berlin, Germany (1987).

11. MATLAB version 5.2 (1998), The MathWorks, Inc

12. J. Bauer, G. Drescher, and M. Illig, "Surface tension, adhesion, and wetting of materials for photolithographic process," J. Vac. Sci. Technol. B 14, 2485-2492 (1996).

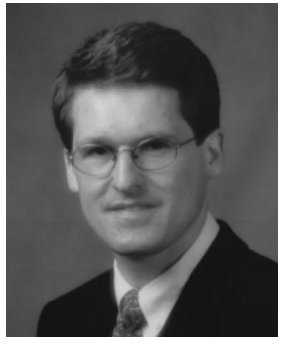

Andreas Schilling received his MS degree from Portland State University, USA, in 1994 during a student exchange program and graduated with a diploma in physics from the University of Konstanz, Germany, in 1996. Subsequently, he joined the Applied Optics Group at the Institute of Microtechnology in Neuchâtel, Switzerland, where he is currently working toward a $\mathrm{PhD}$ as a research assistant in the field of diffractive optics and micro-optics.

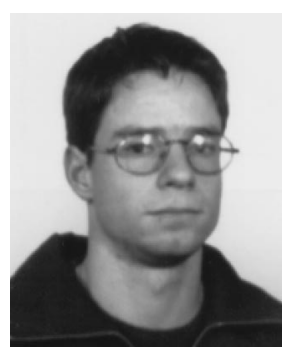

Roman Merz is a graduate student in physical electronics at the Institute of $\mathrm{Mi}$ crotechnology, University of Neuchâtel, Switzerland, since 1996. He is currently working in the field of image processing towards his diploma.



Christian Ossmann received his diploma in 1996 from the Technical University Braunschweig, Germany. Starting with his diploma thesis he worked on LIGA based microlenses at the Institute of Microstructure Technology at the Research Center Karlsruhe, Germany. He joined the Applied Optics Group at the Institute of Microtechnology in Neuchatel, Switzerland, in 1997. There he worked till December 1998 in the field of microlens technology, especially on Microlens Projection Lithography. Since 1999 he works for Karl Suss Neuchatel as head of micro-optics fabrication.

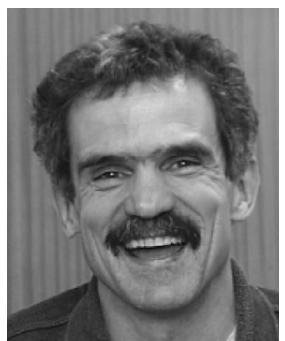

Hans Peter Herzig received his diploma in physics from the Swiss Federal Institute of Technology in Zürich, Switzerland, in 1978. From 1978 to 1982 he was a scientist with the Optics Development Department of Kern in Aarau, Switzerland, working in lens design and optical testing. In 1983, he became a graduate research assistant with the Applied Optics Group at the Institute of Microtechnology of the University of Neuchâtel, Switzerland, working in the field of holographic optical elements, especially scanning elements. In 1987, he received his PhD degree in optics. He currently heads the micro-optics research group and is an associate professor at the University of Neuchâtel. Dr. Herzig is member of OSA and EOS and a board member of the Swiss Society of Optics and Microscopy. 